\title{
SOME RANDOM APPROXIMATIONS AND RANDOM FIXED POINT THEOREMS FOR 1-SET-CONTRACTIVE RANDOM OPERATORS
}

\author{
LIU LI-SHAN
}

(Communicated by Palle E. T. Jorgensen)

\begin{abstract}
In this paper, we will prove that the random version of Fan's Theorem (Math. Z. 112 (1969), 234-240) is true for 1-set-contractive random operator $f: \Omega \times B_{R} \rightarrow X$, where $B_{R}$ is a weakly compact separable closed ball in a Banach space $X$ and $\Omega$ is a measurable space. This class of 1 -set-contractive random operator includes condensing random operators, semicontractive random operators, $L A N E$ random operators, nonexpansive random operators and others. As applications of our theorems, some random fixed point theorems of non-self-maps are proved under various well-known boundary conditions.
\end{abstract}

\section{INTRODUCTION AND PRELIMINARIES}

Since Bharucha-Reid [1] proved the stochastic version of the well-known Schauder's fixed point theorem, random fixed point theory and applications have been developed rapidly in recent years (see, e.g., $[5,6,8,9,13,15,16])$. In this paper, we will consider a stochastic version of a very interesting theorem of Fan [4, Theorem 2] which is stated as follows:

Let $K$ be a nonempty compact convex set in a normed linear space $X$. For any continuous map $f$ from $K$ into $X$, there exists a point $u \in K$ such that

$$
\|u-f(u)\|=d(f(u), K) .
$$

This theorem has been of great importance in Nonlinear Analysis, Approximation Theory, Game Theory and Minimax Theorems. Various aspects of the above theorem have been studied by Lin [7, 8], Papageorgiou [13], Sehgal and Singh [15], Sehgal and Waters [16], and others. Recently, Lin [8, Theorem 1] proved that Ky Fan's Theorem is true for a continuous condensing random operator $f: \Omega \times B_{R} \rightarrow X$, where $B_{R}$ is a separable closed ball in a Banach space $X$ and $\Omega$ is a measurable space. The purpose of the present paper is to extend Lin's result to more general 1-set-contractive random operators in a Banach space. We will also prove that the random version of the above theorem is true for a semicontractive (or $L A N E$ ) random operator $f: \Omega \times B_{R} \rightarrow X$, where $B_{R}$ is a separable closed ball in a uniformal convex Banach space. As applications of our theorems, some stochastic fixed point theorems are derived under various well-known boundary conditions.

Received by the editors August 30, 1994 and, in revised form, August 22, 1995

1991 Mathematics Subject Classification. Primary 47H10, 60H25; Secondary 41A50.

Key words and phrases. Random approximations and random fixed point theorems, 1-setcontractive random operator, semicontractive random operator, $L A N E$ random operators.

(C)1997 American Mathematical Society 
Throughout this paper, $(\Omega, \Sigma)$ denotes a measurable space with $\Sigma$ a sigma algebra of subsets of $\Omega$. Let $X$ be a Banach space. A map $F: \Omega \rightarrow X$ is said to be measurable (respectively, weakly measurable) if $F^{-1}(B)=\{\omega \in \Omega: F(\omega) \in B\}$ $\in \Sigma$ for each closed (respectively, open) subset $B$ of $X$. Let $D$ be a nonempty subset of $X$. Then a map $f: \Omega \times D \rightarrow X$ is called a random operator if for each fixed $x \in D$, the map $f(\cdot, x): \Omega \rightarrow x$ is measurable. A measurable map $\varphi: \Omega \rightarrow D$ is called a random fixed point of $f$ if $f(\omega, \varphi(\omega))=\varphi(\omega)$ for each $\omega \in \Omega$.

A map $f: D \rightarrow X$ is called nonexpansive if $\|f(x)-f(y)\| \leq\|x-y\|$ for $x$, $y \in D ; f$ is called completely continuous if it maps weakly convergent sequences into strongly convergent sequences. We recall that a map $f: D \rightarrow X$ is said to be demiclosed at $y \in X[3]$ if, for any sequence $\left\{x_{n}\right\}$ in $D$, the conditions $x_{n} \rightarrow x \in D$ weakly and $f\left(x_{n}\right) \rightarrow y$ strongly imply $f(x)=y$. A map $f: D \rightarrow X$ is called a $k$ set-contractive [14] $(k \geq 0)$ if $f$ is continuous and, for each bounded subset $B$ of $D$, $\alpha(f(B)) \leq k \alpha(B)$, where $\alpha(\cdot)$ denotes Kuratowski's measure of noncompactness; $f: D \rightarrow X$ is said to be condensing if $f$ is continuous and, for each bounded subset $B$ of $D$ with $\alpha(B)>0, \alpha(f(B))<\alpha(B)$. A map $f: D \rightarrow X$ is said to be semicontractive $[3,14]$ if there exists a map $V$ of $D \times D$ into $X$ such that $f(x)=V(x, x)$ for $x \in D$, while:

(a) For each fixed $x$ in $D, V(\cdot, x)$ is nonexpansive from $D$ to $X$.

(b) For each fixed $x$ in $D, V(x, \cdot)$ is completely continuous from $D$ to $X$, uniformly for $x$ in bounded subset of $D$.

We say that a continuous map $f$ of $D$ into $X$ is $L A N E$ [11] (locally almost nonexpansive) if given $x \in D$ and $\epsilon>0$, there exists a weak neighborhood $N_{x}$ of $x$ in $D$ (depending also on $\epsilon$ ) such that $\|f(u)-f(v)\| \leq\|u-v\|+\epsilon$ for $u, v \in N_{x}$.

A random operator $f: \Omega \times D \rightarrow X$ is said to be continuous (1-set-contractive, condensing, nonexpansive, semicontractive, $L A N E$, completely continuous, etc.) if the map $f(\omega, \cdot): D \rightarrow X$ is so, for each $\omega \in \Omega$. For $R>0$, let

$$
B_{R}=\{x \in X:\|x\| \leq R\}, \quad S_{R}=\{x \in X:\|x\|=R\} .
$$

\section{MAIN RESULtS}

In order to prove our main theorems we need the following lemma which will play a crucial role in this paper.

Lemma 2.1. Let $X$ be a Banach space and $T: B_{R} \rightarrow X$ be a 1-set-contractive map such that $I-T$ is demiclosed at 0 , where $I$ is the identity map on $X$. Then $I-B$ is also demiclosed at 0 , where $B \equiv h T: B_{R} \rightarrow B_{R}$, and $h: X \rightarrow B_{R}$ is a map defined by

$$
h(x)= \begin{cases}x & \text { if }\|x\| \leq R, \\ \frac{R x}{\|x\|} & \text { if }\|x\| \geq R .\end{cases}
$$

Proof. Let $\left\{x_{n}\right\}$ be any sequence in $B_{R}$ such that

$$
x_{n} \rightarrow u \in B_{R} \text { weakly, } \quad x_{n}-B\left(x_{n}\right) \rightarrow 0 \quad \text { as } n \rightarrow \infty .
$$

We have to distinguish two possible cases:

Case 1. If there exists a subsequence $\left\{x_{n_{k}}\right\}$ of $\left\{x_{n}\right\}$ such that $\left\|T\left(x_{n_{k}}\right)\right\| \leq R$ for all $k$, then $B\left(x_{n_{k}}\right)=T\left(x_{n_{k}}\right)$ by the definition of $h$. Hence, $x_{n_{k}}-T\left(x_{n_{k}}\right)=x_{n_{k}}-$ $B\left(x_{n_{k}}\right) \rightarrow 0$ as $k \rightarrow \infty$. It follows from the demiclosedness at 0 of $I-T$ that $u-T(u)=0$. Therefore, $B(u)=h(T(u))=h(u)=u$ by the definition of $h$. 
Case 2. Otherwise, there exists an integer $N$ such that, for $n \geq N,\left\|T\left(x_{n}\right)\right\|>R$, then $B\left(x_{n}\right)=R T\left(x_{n}\right) /\left\|T\left(x_{n}\right)\right\|$ by the definition of $h$. Since $T$ is a 1 -set-contractive map and $\left\{x_{n}\right\}$ is bounded, $\left\{\left\|T\left(x_{n}\right)\right\|\right\}$ is bounded. Hence there exist a convergent subsequence $\left\{\left\|T\left(x_{n_{i}}\right)\right\|\right\}$ of $\left\{\left\|T\left(x_{n}\right)\right\|\right\}$ and a real number $r>0$ such that

$$
\left\|T\left(x_{n_{i}}\right)\right\| \rightarrow r \quad \text { as } i \rightarrow \infty .
$$

From $\left\|T\left(x_{n_{i}}\right)\right\|>R(i=1,2,3, \cdots)$, we have $r \geq R$. Therefore, by (2.1) and (2.2), we have

$$
\begin{aligned}
x_{n_{i}}-\frac{R}{r} T\left(x_{n_{i}}\right) & =\left(x_{n_{i}}-\frac{R T\left(x_{n_{i}}\right)}{\left\|T\left(x_{n_{i}}\right)\right\|}\right)+\left(\frac{R T\left(x_{n_{i}}\right)}{\left\|T\left(x_{n_{i}}\right)\right\|}-\frac{R}{r} T\left(x_{n_{i}}\right)\right) \\
& =\left(x_{n_{i}}-B\left(x_{n_{i}}\right)\right)+\left(\frac{1}{\left\|T\left(x_{n_{i}}\right)\right\|}-\frac{1}{r}\right) R T\left(x_{n_{i}}\right) \\
& \rightarrow 0 \text { as } i \rightarrow \infty .
\end{aligned}
$$

If $r=R$, then $x_{n_{i}}-T\left(x_{n_{i}}\right) \rightarrow 0(i \rightarrow \infty)$, by (2.3). It follows from the demiclosedness at 0 of $I-T$ that $u=T(u)$. Therefore, $B(u)=h(T(u))=h(u)=u$ by the definition of $h$. If $r>R$, then $(R / r) T$ is a $(R / r)$-set-contractive map with $R / r<1$. Let $y_{n_{i}} \equiv x_{n_{i}}-(R / r) T\left(x_{n_{i}}\right)$. Then $\alpha\left(\left\{y_{n_{i}}\right\}\right)=0$ and $x_{n_{i}}=y_{n_{i}}+(R / r) T\left(x_{n_{i}}\right)$. It follows that $\alpha\left(\left\{x_{n_{i}}\right\}\right)=0$. Hence there exists a subsequence $\left\{x_{n_{i_{j}}}\right\}$ of $\left\{x_{n_{i}}\right\}$ such that $\left\{x_{n_{i_{j}}}\right\}$ converges to $u$. This and the continuity of $T$ imply that $y_{n_{i_{j}}} \rightarrow$ $u-(R / r) T(u)=0$, i.e., $u=(R / r) T(u)$. From $(2.2)$, we have $\|T(u)\|=r>R$. Therefore $u=R T(u) /\|T(u)\|=B(u)$ by the definition of $h$.

This completes the proof of Lemma 2.1.

We shall also need the following random fixed point theorem which is Theorem 2.1 in [9]:

Lemma 2.2. Let $D$ be a nonempty weakly compact convex subset of a separable Banach space $X$, and $f: \Omega \times D \rightarrow D$ be a 1-set-contractive random operator such that $I-f(\omega, \cdot)$ is demiclosed, for each $\omega \in \Omega$. Then $f$ has a random fixed point.

Remark 2.1. From the proof of Lin's theorem above, we can easily see that we only need the hypothesis $I-f(\omega, \cdot)$ to be demiclosed at 0 for each $\omega \in \Omega$ instead of $I-f(\omega, \cdot)$ demiclosed for each $\omega \in \Omega$, and Lin's result also holds, if we assume that $D$ is separable instead of $X$ is separable.

Theorem 2.1. Let $B_{R}$ be a weakly compact separable subset of $X$ and $f: \Omega \times B_{R} \rightarrow$ $X$ be a 1-set-contractive random operator such that $I-f(\omega, \cdot)$ is demiclosed at 0 , for each $\omega \in \Omega$, where $I$ is the identity map on $X$. Then there exists a measurable map $\varphi: \Omega \rightarrow B_{R}$ such that for each $\omega \in \Omega$

$$
\|\varphi(\omega)-f(\omega, \varphi(\omega))\|=d\left(f(\omega, \varphi(\omega)), B_{R}\right) .
$$

Moreover, to this $\varphi$, for each $\omega \in \Omega$, if $\|f(\omega, \varphi(\omega))\|>R$, then $\varphi(\omega) \in S_{R}$ and

$$
\varphi(\omega)=\frac{R f(\omega, \varphi(\omega))}{\|f(\omega, \varphi(\omega))\|}, \quad\|f(\omega, \varphi(\omega))-\varphi(\omega)\|=\|f(\omega, \varphi(\omega))\|-R>0
$$

if $\|f(\omega, \varphi(\omega))\| \leq R$, then $\varphi(\omega)=f(\omega, \varphi(\omega))$.

Proof. Let $h$ be the same as in Lemma 2.1. From Nussbaum [10, Corollary 1], $h$ is a 1-set-contractive map. Define $F: \Omega \times B_{R} \rightarrow B_{R}$ by $F(\omega, x)=h(f(\omega, x))$. It is easy to see that $F$ is a 1 -set-contractive random operator. For arbitrary but fixed 
$\omega \in \Omega, I-F(\omega, \cdot)$ is demiclosed at 0 by Lemma 2.1. From Lemma 2.2, $F$ has a random fixed point $\varphi: \Omega \rightarrow B_{R}$, i.e., $\varphi$ is a measurable map and $F(\omega, \varphi(\omega))=\varphi(\omega)$ for each $\omega \in \Omega$. Now we will prove that this measurable map $\varphi$ satisfies the desired property. For each $\omega \in \Omega$, we consider the following two cases:

(i) If $\|f(\omega, \varphi(\omega))\| \leq R$, then $F(\omega, \varphi(\omega))=f(\omega, \varphi(\omega))$ by the definition of $h$. Thus, we have $\|\varphi(\omega)-f(\omega, \varphi(\omega))\|=\|F(\omega, \varphi(\omega))-f(\omega, \varphi(\omega))\|=0$.

(ii) If $\|f(\omega, \varphi(\omega))\|>R$, then $F(\omega, \varphi(\omega))=R f(\omega, \varphi(\omega)) /\|f(\omega, \varphi(\omega))\|$ by the definition of $h$. Hence, we have

$$
\begin{aligned}
\|\varphi(\omega)-f(\omega, \varphi(\omega))\| & =\|F(\omega, \varphi(\omega))-f(\omega, \varphi(\omega))\| \\
& =\left\|\frac{R f(\omega, \varphi(\omega))}{\|f(\omega, \varphi(\omega))\|}-f(\omega, \varphi(\omega))\right\| \\
& =\|f(\omega, \varphi(\omega))\|-R .
\end{aligned}
$$

Thus, for any $x \in B_{R}$, we have

$$
\begin{aligned}
\|\varphi(\omega)-f(\omega, \varphi(\omega))\| & \leq\|f(\omega, \varphi(\omega))\|-R \\
& \leq\|f(\omega, \varphi(\omega))\|-\|x\| \\
& \leq\|f(\omega, \varphi(\omega))-x\| .
\end{aligned}
$$

Therefore, (2.4) holds.

For each $\omega \in \Omega$, if $\|f(\omega, \varphi(\omega))\|>R$ with $\varphi(\omega) \in B_{R}$, then $f(\omega, \varphi(\omega)) \notin B_{R}$ and by (ii), (2.5) holds. If $\|\varphi(\omega)\|<R$, then there exists $a \in(0,1)$ such that $u \equiv a \varphi(\omega)+(1-a) f(\omega, \varphi(\omega)) \in B_{R}$. It follows that

$$
\begin{aligned}
d\left(f(\omega, \varphi(\omega)), B_{R}\right) & \leq\|f(\omega, \varphi(\omega))-u\| \\
& =a\|f(\omega, \varphi(\omega))-\varphi(\omega)\| \\
& <\|f(\omega, \varphi(\omega))-\varphi(\omega)\| \\
& =d\left(f(\omega, \varphi(\omega)), B_{R}\right) .
\end{aligned}
$$

We get a contradiction. Hence $\varphi(\omega) \in S_{R}$. If $\|f(\omega, \varphi(\omega))\| \leq R$ with $\varphi(\omega) \in B_{R}$, then by (i) we have $\varphi(\omega)=f(\omega, \varphi(\omega))$.

This completes the proof of the theorem.

Theorem 2.2. Let $B_{R}$ be a separable subset of a uniformly convex Banach space $X$ and $f: \Omega \times B_{R} \rightarrow X$ be a semicontractive random operator. Then the conclusions of Theorem 2.1 hold.

Proof. From Petryshyn [14, Lemma 3.2 and p. 338], $f: \Omega \times B_{R} \rightarrow X$ is 1-setcontractive random operator. By Browder [3, Theorem 3], $I-f(\omega, \cdot)$ is demiclosed, for each $\omega \in \Omega$. Hence, Theorem 2.2 follows from Theorem 2.1 .

Corollary 2.1. Let $B_{R}$ be a separable subset of a uniformly convex Banach space $X, g: \Omega \times B_{R} \rightarrow X$ a nonexpansive random operator and $h: \Omega \times B_{R} \rightarrow X$ a completely continuous random operator. If $f \equiv g+h$, then the conclusions of Theorem 2.1 hold.

Proof. Since $f: \Omega \times B_{R} \rightarrow X$ is a semicontractive random operator under the representation $V(\omega, u, v)=g(\omega, u)+h(\omega, v)$, this corollary just follows from Theorem 2.2 .

Theorem 2.3. Let $B_{R}$ be a separable subset of a uniformly convex Banach space $X, g: \Omega \times B_{R} \rightarrow X$ a LANE random operator, and $h: \Omega \times B_{R} \rightarrow X$ a completely 
continuous random operator. If $f \equiv g+h$, then the conclusions of Theorem 2.1 hold.

Proof. From [14, Remark 3.7], $f: \Omega \times B_{R} \rightarrow X$ is also a $L A N E$ random operator. By [11], $f$ is a 1-set-contractive random operator and $I-f(\omega, \cdot)$ is demiclosed, for each $\omega \in \Omega$. Therefore, Theorem 2.3 follows from Theorem 2.1.

The following is another random approximation theorem of Ky Fan type. Before we state the theorem, we recall a definition. A Banach space $X$ is said to satisfy Opial's condition [12] if the following holds: If $\left\{x_{n}\right\}$ converges weakly to $x_{0}$, and $x \neq x_{0}$, then liminf $\left\|x_{n}-x\right\|>\liminf \left\|x_{n}-x_{0}\right\|$. Banach spaces satisfying Opial's condition include Hilbert spaces and $l^{p} \quad(1 \leq p<\infty)$ spaces.

Theorem 2.4. Let $B_{R}$ be a weakly compact separable closed ball of a Banach space $X, g: \Omega \times B_{R} \rightarrow X$ a nonexpansive random operator, and $h: \Omega \times B_{R} \rightarrow X$ a completely continuous random operator. If $X$ satisfies Opial's condition and $f \equiv g+h$, then the conclusions of Theorem 2.1 hold.

Proof. Since $f$ is 1-set-contractive and $I-f(\omega, \cdot)$ is demiclosed, for each $\omega \in \Omega$, by Opial [12] if $X$ satisfies Opial's condition, Theorem 2.4 follows from Theorem 2.1 .

\section{ApPlichtions to RANDOM FIXED POINT THEOREMS}

Theorem 3.1. Suppose that $X, B_{R}$ and $f$ are the same as in Theorem 2.1. Then $f$ has a random fixed point if $f$ satisfies one of the following conditions:

$\left(\mathrm{B}_{1}\right)$ For each $\omega \in \Omega$, each $x \in S_{R}$ with $\|f(\omega, x)\|>R$, there exists $y$, depending on $\omega$ and $x$, in $I_{B_{R}}(x)=\left\{x+c(z-x)\right.$ : some $\left.z \in B_{R}, c>0\right\}$ such that $\| y-$ $f(\omega, x)\|<\| x-f(\omega, x) \|$.

$\left(\mathrm{B}_{2}\right) f$ is weakly inward, i.e., for each $\omega \in \Omega, f(\omega, x) \in \overline{I_{B_{R}}(x)}$ for $x \in S_{R}$.

$\left(\mathrm{B}_{3}\right) x \neq \lambda f(\omega, x)$ for each $\omega \in \Omega$ and $x \in S_{R}$ with $\|f(\omega, x)\|>R$ and $0<\lambda<1$. $R$.

$\left(\mathrm{B}_{4}\right)\|f(\omega, x)-x\| \neq\|f(\omega, x)\|-R$, for each $\omega \in \Omega$ and $x \in S_{R}$ with $\|f(\omega, x)\|>$

$\left(\mathrm{B}_{5}\right)$ For each $\omega \in \Omega$ and $x \in S_{R}$, with $\|f(\omega, x)\|>R$, there exists $\alpha \in(1, \infty)$ such that

$$
\|f(\omega, x)\|^{\alpha}-R^{\alpha} \leq\|f(\omega, x)-x\|^{\alpha} .
$$

$\left(\mathrm{B}_{6}\right)$ For each $\omega \in \Omega$ and $x \in S_{R}$, with $\|f(\omega, x)\|>R$, there exists $\beta \in(0,1)$ such that

$$
\|f(\omega, x)\|^{\beta}-R^{\beta} \geq\|f(\omega, x)-x\|^{\beta} .
$$

Proof. By Theorem 2.1, there exists a measurable map $\varphi: \Omega \rightarrow B_{R}$ such that, for each $\omega \in \Omega$, (2.4) holds, and for each $\omega \in \Omega$, if $\|f(\omega, \varphi(\omega))\|>R$, then $\|\varphi(\omega)\|=R$ and (2.5) holds; if $\|f(\omega, \varphi(\omega))\| \leq R$, then $\varphi(\omega)=f(\omega, \varphi(\omega))$. We will prove that $\varphi$ is the desired random fixed point of $f$. Toward this end, we consider the following two cases:

(a) If there exists $\omega \in \Omega$, such that $\|\varphi(\omega)\|<R$, then there exists $\lambda \in(0,1)$ such that $\|\lambda \varphi(\omega)+(1-\lambda) f(\omega, \varphi(\omega))\|<R$. Therefore

$$
\begin{aligned}
& \|\varphi(\omega)-f(\omega, \varphi(\omega))\|=d\left(f(\omega, \varphi(\omega)), B_{R}\right) \\
& \quad \leq\|f(\omega, \varphi(\omega))-[\lambda \varphi(\omega)+(1-\lambda) f(\omega, \varphi(\omega))]\|=\lambda\|\varphi(\omega)-f(\omega, \varphi(\omega))\|,
\end{aligned}
$$

it follows from $0<\lambda<1$ that $\varphi(\omega)=f(\omega, \varphi(\omega))$. 
(b) If there exists $\omega \in \Omega$, such that $\|\varphi(\omega)\|=R$, we will show that $\varphi(\omega)=$ $f(\omega, \varphi(\omega))$. By $(2.4)$, we need only show that $f(\omega, \varphi(\omega)) \in B_{R}$.

(1) If $\|f(\omega, \varphi(\omega))\|>R$ and $f$ satisfies $\left(\mathrm{B}_{1}\right)$, then there exists $y$ in $I_{B_{R}}(\varphi(\omega))$ such that

$$
\|y-f(\omega, \varphi(\omega))\|<\|\varphi(\omega)-f(\omega, \varphi(\omega))\| .
$$

Since $y \in I_{B_{R}}(\varphi(\omega))$, there exists $z \in B_{R}, c>0$ such that $y=\varphi(\omega)+$ $c(z-\varphi(\omega))$. Since $y \notin B_{R}$ (otherwise it contradicts the choice of $\varphi$ ), we can assume $c>1$. Then

$$
z=\frac{y}{c}+\left(1-\frac{1}{c}\right) \varphi(\omega)=(1-\beta) y+\beta \varphi(\omega),
$$

where $\beta=1-1 / c, 0<\beta<1$. Therefore

$$
\begin{aligned}
\|z-f(\omega, \varphi(\omega))\| & \leq(1-\beta)\|y-f(\omega, \varphi(\omega))\|+\beta\|\varphi(\omega)-f(\omega, \varphi(\omega))\| \\
& <(1-\beta)\|\varphi(\omega)-f(\omega, \varphi(\omega))\|+\beta\|\varphi(\omega)-f(\omega, \varphi(\omega))\| \\
& =\|\varphi(\omega)-f(\omega, \varphi(\omega))\|,
\end{aligned}
$$

which contradict the choice of $\varphi$. Hence $f(\omega, \varphi(\omega)) \in B_{R}$.

(2) If $f$ satisfies condition $\left(\mathrm{B}_{2}\right)$, then $f$ satisfies $\left(\mathrm{B}_{1}\right)$.

(3) If $\|f(\omega, \varphi(\omega))\|>R$ and $f$ satisfies $\left(\mathrm{B}_{3}\right)$, then by $(2.5)$ we have $\varphi(\omega)=$ $\lambda_{0} f(\omega, \varphi(\omega))$, where $\lambda_{0}=R /\|f(\omega, \varphi(\omega))\|, 0<\lambda_{0}<1$, which contradicts condition $\left(\mathrm{B}_{3}\right)$. Therefore, $f(\omega, \varphi(\omega)) \in B_{R}$.

(4) If $\|f(\omega, \varphi(\omega))\|>R$ and $f$ satisfies $\left(\mathrm{B}_{4}\right)$, then by $(2.5)$ we have $\| f(\omega, \varphi(\omega))-$ $\varphi(\omega)\|=\| f(\omega, \varphi(\omega)) \|-R$. This is a contradiction to the condition $\left(\mathrm{B}_{4}\right)$. Therefore, $f(\omega, \varphi(\omega)) \in B_{R}$. that

(5) If $\|f(\omega, \varphi(\omega))\|>R$ and $f$ satisfies $\left(B_{5}\right)$, then there exists $\alpha \in(1, \infty)$ such

$$
\|f(\omega, \varphi(\omega))\|^{\alpha}-R^{\alpha} \leq\|f(\omega, \varphi(\omega))-\varphi(\omega)\|^{\alpha} .
$$

Let $\lambda=R /\|f(\omega, \varphi(\omega))\|$; then $0<\lambda<1$ and

$$
\frac{(\|f(\omega, \varphi(\omega))\|-R)^{\alpha}}{\|f(\omega, \varphi(\omega))\|^{\alpha}}=(1-\lambda)^{\alpha}<1-\lambda^{\alpha} \leq \frac{\|f(\omega, \varphi(\omega))-\varphi(\omega)\|^{\alpha}}{\|f(\omega, \varphi(\omega))\|^{\alpha}} .
$$

Hence $\|f(\omega, \varphi(\omega))-\varphi(\omega)\|>\|f(\omega, \varphi(\omega))\|-R$, we get a contradiction to (2.5). Therefore, $f(\omega, \varphi(\omega)) \in B_{R}$.

(6) If $\|f(\omega, \varphi(\omega))\|>R$ and $f$ satisfies $\left(\mathrm{B}_{6}\right)$, then similar to the proof of (5), we can prove that $f(\omega, \varphi(\omega)) \in B_{R}$.

In sum, we have shown that the measurable map $\varphi: \Omega \rightarrow B_{R}$ satisfies $\varphi(\omega)=$ $f(\omega, \varphi(\omega))$ for each $\omega \in \Omega$, i.e., $\varphi$ is a random fixed point of $f$.

Using similar methods, we can show the following theorems and corollary, and we omit their proof.

Theorem 3.2. Let $B_{R}$ be a separable subset of a uniformly convex Banach space $X$. If $f: \Omega \times B_{R} \rightarrow X$ is a semicontractive random operator and satisfies any one of the six conditions of Theorem 3.1, then $f$ has a random fixed point.

Corollary 3.1. Let $B_{R}$ be a separable subset of a uniformly convex Banach space $X, g: \Omega \times B_{R} \rightarrow X$ a nonexpansive random operator, and $h: \Omega \times B_{R} \rightarrow X a$ completely continuous random operator. If $f \equiv g+h$ satisfies any one of the six conditions of Theorem 3.1, then $f$ has a random fixed point. 
Theorem 3.3. Let $B_{R}$ be a separable subset of a uniformly convex Banach space $X, g: \Omega \times B_{R} \rightarrow X$ a LANE random operator, and $h: \Omega \times B_{R} \rightarrow X$ a completely continuous random operator. If $f \equiv g+h$ satisfies any one of the six conditions of Theorem 3.1, then $f$ has a random fixed point.

Theorem 3.4. Let $B_{R}$ be a weakly compact separable closed ball of a Banach space $X, g: \Omega \times B_{R} \rightarrow X$ a nonexpansive random operator, and $h: \Omega \times B_{R} \rightarrow X$ a completely continuous random operator. If $X$ satisfies Opial's condition and $f \equiv g+h$ satisfies any one of the six conditions of Theorem 3.1, then $f$ has a random fixed point.

\section{ACKNOWLEDGMENT}

The author would like to thank the referee for reading this paper carefully, providing valuable suggestions and comments, and pointing out a major error in the original version of this paper.

\section{REFERENCES}

1. A. T. Bharucha-Reid, Fixed point theorems in probabilistic analysis, Bull. Amer. Math. Soc. 82 (1976), 641-645. MR 54:1390

2. A. T. Bharucha-Reid, Random integral equations, Academic Press, New York and London, 1972. MR 56:1459

3. F. E. Browder, Semicontractive and semiaccretive nonlinear mappings in Banach spaces, Bull. Amer. Math. Soc. 74 (1968), 660-665. MR 37:5742

4. K. Fan, Extensions of two fixed point theorems of F. E. Browder, Math. Z. 112 (1969), 234-240. MR 40:4830

5. S. Itoh, Random fixed point theorems with an application to random differential equations in Banach spaces, J. Math. Anal. Appl. 67 (1979), 261-273. MR 80f:60059

6. K. Kuratowski and C. Ryll-Nardzewski, A general theorem on selector, Bull. Acad. Polon. Sci. Ser. Sci. Math. Astronom. Phys. 13 (1965), 397-403. MR 32:6421

7. T. C. Lin, A note on a theorem of Ky Fan, Canad. Math. Bull. 22 (1979), 513-515. MR 81d: 47038

8. T. C. Lin, Random approximations and random fixed point theorems for non-self-maps, Proc. Amer. Math. Soc. 103 (1988), 1129-1135. MR 89i:47109

9. T. C. Lin, Random approximations and random fixed point theorems for continuous 1-setcontractive random maps, Proc. Amer. Math. Soc. 123 (1995), 1167-1176. MR 95e:47088

10. R. D. Nussbaum, The fixed point index for local condensing maps, Ann. Mat. Pura Appl. 89 (1971), 217-258. MR 47:903

11. R. D. Nussbaum, The fixed point index and fixed point theorems for $k$-set-contractions, Ph. D. Thesis, Univ. of Chicago (1969).

12. Z. Opial, Weak convergence of the successive approximations for nonexpansive mappings, Bull. Amer. Math. Soc. 73 (1967), 591-597. MR 35:2183

13. N. S. Papageorgiou, Random fixed point of theorems for measurable multifunctions in Banach spaces, Proc. Amer. Math. Soc. 97 (1986), 507-514. MR 88a:60117.

14. W. V. Petryshyn, Fixed point theorems for various classes of 1-set-contractive and 1-ballcontractive mappings in Banach spaces, Trans. Amer. Math. Soc. 182 (1973), 323-352. MR 48:7030

15. V. M. Sehgal and S. P. Singh, On random approximations and a random fixed point theorem for set valued mappings, Proc. Amer. Math. Soc. 95 (1985), 91-94. MR 86k:47049

16. V. M. Sehgal and C. Waters, Some random fixed point theorems for condensing operators, Proc. Amer. Math. Soc. 90 (1984), 425-429. MR 85g:47083

Department of Mathematics, Qufu Normal University, Qufu, Shandong, 273165, PeoPle's RePublic of China 